\title{
Unwetterkatastrophe: Versicherungspflicht sinnvoll!
}

Die jüngste Flutkatastrophe hat klar gemacht: Wir brauchen mehr baurechtliche und technische Vorsorge, um mit Wetterextremen besser zurecht zu kommen. Aber auch künftige Bauverbote in extrem von Hochwasser gefährdeten Lagen und weniger Bodenversiegelung werden alles andere als hundertprozentig vor Starkregen, Hochwasser, Sturm oder Schneedruck schützen. Zumal wir seit Jahrhunderten bebaute Liegenschaften nicht „entsiedeln“ können - dies geht noch nicht einmal für erst in jüngster Zeit mit unsinnigen Baugenehmigungen errichtete neue Wohngebäude in Überschwemmungsgegenden. Auch die Eigentümer:innen solcher Immobilien haben ein Recht auf Schutz, d. h. auch auf Versicherungsschutz, der allerdings im Moment in Hochrisikolagen nur schwer zu erlangen ist.

Um die Resilienz zu steigern, also die Fähigkeit sich nach einer Krise möglichst rasch zu erholen, ist eine gesetzliche Pflicht zur Versicherung von Wohngebäuden gegen Naturgefahren sinnvoll. Und eine risikogerechte Versicherung wirkt zweifach: Sie sichert nicht nur im Schadensfall planmäßig ab (steigert also die Resilienz), sondern die Prämien für die Versicherung stellen auch einen steten Anreiz dar, individuelle und gesellschaftliche Vorsorge zu betreiben und so die Schäden durch extreme Wetterereignisse möglichst klein zu halten. Dafür müssen die Prämien je nach Gefahrenlage und Vorsorge gestaffelt sein. Niemand zahlt gerne immer höhere Versicherungsprämien, und Tote werden dadurch auch nicht wieder lebendig, aber durch individuelle und gesellschaftliche Vorsorge kann es künftig weniger Klimatote und Klimaschäden geben. Darauf weist auch die Nationalakademie Leopoldina im Rahmen einer aktuellen Stellungnahme zur Corona-Pandemie hin: „Neben Pandemien sind Flut- und Dürreprobleme klimabedingte Naturphänomene, für die Vorsorge getroffen werden kann." Zur Vorsorge können entsprechende Versicherungen gehören, und eine solche „Ex-ante-Absicherung kann nicht nur zu einem besseren Schutz nach Eintritt eines Schadenfalls beitragen, sondern auch zu zusätzlichen präventiven Maßnahmen zur Minimierung möglicher Schäden führen und so die gesellschaftliche Resilienz erhöhen“.

Unwetterschäden sind in Deutschland mit einer Elementarschadenversicherung im Rahmen einer Wohngebäudeversicherung grundsätzlich versicherbar. Nach Berechnungen des Sachverständigenrats für Verbraucherfragen (SVRV) lassen sich gegenwärtig noch die meisten Wohngebäude sogar für nur 5 Euro im Monat bei minimalem Selbstbehalt im Schadensfall versichern. Selbst in Regionen mit hoher Unwettergefahr sind Wohngebäude versicherbar, wenn hinreichend technische Vorsorge betrieben wird (und z. B. die Keller nicht als Wohnraum genutzt werden) und ein gewisser Selbstbehalt akzeptiert wird, der häufig vorkommende kleine und mittlere Schäden, die Hausund Wohnungseigentümer:innen selbst tragen können, nicht versichert. Die Mehrheit der Hausbesitzenden interessiert sich freilich bislang nicht für geeigneten Versicherungsschutz - das gilt auch für die meisten politischen Parteien. Im Ergebnis sind nur knapp $50 \%$ der Wohngebäude gegen Unwetter versichert. In den nun am stärksten betroffenen Gebieten in Rheinland-Pfalz und im Saarland sind es sogar weniger als $40 \% .2017$ haben sich die Ministerpräsident:innen der Länder darauf verständigt, dass im Katastrophenfall nur noch in Härtefällen an Geschädigte gezahlt werden soll. Die Idee war offenbar, dass durch die bloße Ankündigung der Verweigerung von Hilfe in der Not hinreichend Anreize gesetzt waren, damit die Menschen eigeninitiativ technische

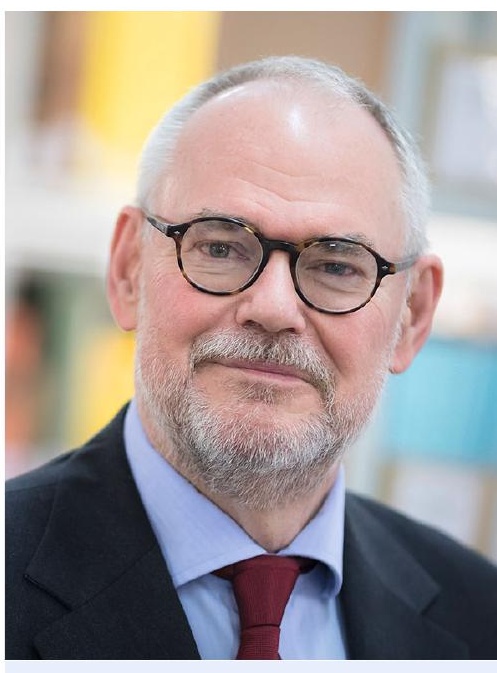

Der Berliner Ökonom Gert G. Wagner ist Mitglied im Sachverständigenrat für Verbraucherfragen (SVRV) und im Sozialbeirat der Bundesregierung. Wagner hat bereits 2002 in Heft 10 des Wirtschaftsdienstes ein Konzept vorgelegt, wie er es hier erneut diskutiert. 
Vorsorgen treffen und eine Elementarschadenversicherung abschließen. Dieses Signal war offenbar wenig glaubwürdig. Und die Menschen sollten Recht behalten mit ihrer Intuition, denn bereits kurz nach der jetzigen katastrophalen Flutwelle wurden finanzielle Hilfen durch den Staat in Aussicht gestellt. Die finanziellen Hilfen sind unter den gegebenen Umständen auch richtig - aber kluge Politik sieht anders aus.

Der SVRV hatte Ende 2019 ein Konzept für eine verpflichtende Elementarschadenversicherung für Haus- und Wohnungseigentümer:innen vorgelegt - kurz danach brach die Corona-Pandemie aus, und das Konzept wurde nicht breit diskutiert. Als eine von mehreren konkreten Maßnahmen schlägt der SVRV vor, dass jedes Wohngebäude in Deutschland verpflichtend gegen katastrophale Naturgefahren mit einer Elementarschadenversicherung versichert sein sollte. Das wird sinnvollerweise nicht mit einem Rundum-Sorglos-Paket erreicht, sondern setzt besonders in höheren Gefahrenlagen bestimmte Vorkehrungen wie Schutzmauern und den technischen Schutz von Lichtschächten voraus, sowie durchaus spürbare Selbstbeteiligungen. Resilienz durch eine Versicherungspflicht für Elementarschäden ist also keineswegs nur ein passives Hinnehmen von Problemen. Denn die Prämien für eine Versicherung gegen Naturgefahren wirken im Ergebnis wie die Bepreisung von Treibhausgasemissionen: Während der $\mathrm{CO}_{2}$-Preis der Umweltschädigung eines Produkts einen Preis gibt, gibt die Versicherungsprämie dem erwartbaren Gebäudeschaden einen Preis. Darauf weisen der SVRV und z. B. die stellvertretende Vorsitzende des Sachverständigenrats für Umweltfragen Claudia Kemfert ausdrücklich hin. Und wer langfristig die Ausgaben für den $\mathrm{CO}_{2}$-Ausstoß niedrig halten möchte oder im Falle der Versicherung gegen Naturgefahren günstige Prämien zahlen will, der muss geeignete Maßnahmen ergreifen, um später einen Vorteil zu haben. Für den Schutz gegen häufig vorkommende Naturgefahren wie Platzregen sind verhältnismäßig günstige technische Maßnahmen, etwa die Installation von Rückschlagklappen beim Abwasserabfluss, oft bereits ausreichend, um schwere Schäden im Keller durch Starkregen zu vermeiden.

Zu Recht wird argumentiert, dass die Gefahren des Klimawandels nicht auf die einzelnen Menschen abgewälzt werden dürfen - dieser Eindruck könnte bei einer individuellen Versicherung gegen Naturgefahren entstehen. Dieser Einwand muss ernst genommen werden. Trotzdem ist die Frage berechtigt, ob die Menschen bessergestellt sind, wenn kaum ein Anreiz besteht, individuelle Vorkehrungen gegen Naturgefahren zu treffen und im Katastrophenfall auf das Wohlwollen des Staates angewiesen zu sein. Wenn die Prämien für eine Elementarschadenversicherung in bestimmten Lagen hunderte Euro pro Jahr für ein Einfamilienhaus betragen würden, könnte der Staat die Last durch einen zielgerichteten Transfer (analog zum Wohngeld) mildern. Denn es ist sinnvoll, dass der Staat die Menschen im Voraus besser schützt als heute. Eine solche Unterstützung sollte vom jeweiligen Bundesland ausgezahlt werden, da die Länder besser als der Bund die örtliche Lage kennen. Wer freilich einen Neubau an einem stark gefährdeten Standort errichtet (sofern dafür künftig noch eine Baugenehmigung erteilt wird), sollte die Versicherungsprämie wie alle anderen Haus- und Wohnungsinhabenden auch voll selbst tragen müssen.

Eine Versicherungspflicht ist auch - wie der SVRV geprüft hat - verfassungsrechtlich zulässig. Wenn man genau hinschaut, ist das auch die Einschätzung der Justizministerkonferenz, die trotzdem noch 2015 die Einführung einer Versicherungspflicht abgelehnt hat. Der Abschlussbericht der Arbeitsgruppe der Justizminister:innen ist zwar noch immer nicht veröffentlicht (Stand: 6. August 2021), aber man hört, dass darin auf das Grünbuch der EU-Kommission „Versicherung gegen Naturkatastrophen und von Menschen verursachte Katastrophen“ verwiesen wird, das feststellt, dass Naturkatastrophen infolge des Klimawandels zunehmen werden. Deswegen sollten die Erfahrun-

Gert G. Wagner

Sachverständigenrat für Verbraucherfragen

gwagner@diw.de gen mit der Bewältigung von Elementarschäden auf globaler und europäischer Ebene regelmäßig evaluiert werden. Je nach Entwicklung der Lage könnte dann auch die Einführung einer Versicherungspflicht erneut geprüft werden. 